\title{
Functional outcomes in acute lateral ankle ligament repair using suture tape augmentation
}

\begin{abstract}
Introduction: Acute lateral ankle injuries are commonly managed conservatively but when repair is done in grade III injuries in the professional athletic population it is repaired using the Broström surgical procedure. Augmentation of this procedure using suture tape has been reported to improve ankle stability and physical patient outcomes.
\end{abstract}

Method: This is a retrospective study of 30 patients with grade II-III anterior talofibular ligament instability, treated with augmented Broström-Gould suture tape procedure.

The patients were followed up a minimum of 14.4 months post-surgery using AOFAS and RAND 36-item Short Form scores. We also evaluated ability and time to return to previous level of activity or sport following surgery and rehabilitation.

Results: Overall patients showed excellent results, with an average AOFAS score of 92.7. According to the SF-36 scale patients perceived low levels of pain (80.6) and good physical functioning (88.3), with $86.7 \%$ of patients in the study being able to return to their previous level of activity or sport on average 10.94 weeks post surgery.

Conclusion: Augmented Broström with suture tape showed excellent AOFAS and favourable RAND SF-36 outcomes in the short term with a good indication of functional outcomes in patients' ability to return to previous levels of activity or sport.

Keywords: internal brace, acute ankle ligament repair, suture tape augmentation
Volume I4 Issue I - 2022

\author{
Ludwig Wilhelm Schmidt,' Jacobus Wessels, ${ }^{2}$ \\ Charl Janse van Rensburg, ${ }^{3}$ Mthunzi \\ Ngcelwane' \\ 'Department of Orthopaedic surgery, University of Pretoria, \\ Pretoria, South Africa \\ 2MMED (Ortho), Netcare Moot Hospital, South Africa \\ ${ }^{3}$ Medical Research Council, Biostatistics Unit, Pretoria, South \\ Africa
}

Correspondence: DR LW Schmidt, University of Pretoria, POBOX 26389, Monumentpark, Pretoria, 0105 ,

Tel +27 (0)I2 354 285 I, Email ludwil.schmidt@gmail.com

Received: December 23, 2021 | Published: January 10, 2022

\section{Introduction}

Ankle sprain is a common sports related injury with estimated 302,000 new cases each year. In the UK, 42,000 of these are considered to be severe injuries. These injuries have a severe impact in the sports industry with players missing training and competition due to injury and recovery time. ${ }^{1}$ The majority of patients recover fully with non-surgical management, but in $10-40 \%$ of patients the injury leads to chronic instability and other permanent sequelae like persistent pain and long term functional limitations like chronic instability or sequela such as osteoarthritis. ${ }^{1-4}$ Surgical management has become more popular in grade and II and III injuries especially for athletes to reduce the recovery time and return to sport and also to prevent the permanent sequelae of chronic ankle instability in the normal population. ${ }^{3}$

The most common direct repair method is the Broström procedure or the modified Broström-Gould procedure. The post-operative patients are then started on a gradual rehabilitation program aiming to return to sport and or previous level of activity within 12 weeks to allow the repair to heal and attain the needed proprioception. Elongation of the anterior talofibular ligament ATFL in unprotected repaired specimens was significantly higher than in the motion protected state. ${ }^{5,6}$

Complete immobilisation even for short periods of time may substantially prolong rehabilitation and return to normal activity. After partial or complete immobility for extended periods of time, more time may be required for the functional capacity of the ligament unit to return to normal. Surgical intervention decreases the chances of patients developing chronic instability and osteoarthritis which can be debilitating requiring salvage procedures like arthrodesis or ankle arthroplasty. Recent advances have allowed repairs to be made with augmentation which strengthens the repair allowing the patient to start earlier rehabilitation programs and earlier return to sport. The brace shows similar biomechanical stability compared to an intact native ATFL in terms of torque and angle at failure. ${ }^{7,8}$ The procedure has good outcomes in biomechanical studies in chronic ligament repairs. ${ }^{89}$ However, limited literature is available for this technique in acute ligament repairs. In this study we aimed to evaluate the functional outcomes in patients with grade two or three acute lateral ankle ligament injuries after modified Broström-Gould procedure with suture tape augmentation.

\section{Methods}

After ethical approval by local ethical review board, we did a retrospective study on all patients who underwent lateral ankle ligament repair with a Broström procedure and Internal Brace ${ }^{T M}$ at a private institution in Pretoria during the period-January 2017 to December 2018.

All consenting participants were evaluated through completion of questionnaires and functional scoring more than 12 months following Broström procedure. Patients that were included in the study had to be older than 18 years, diagnosis of a grade two or three lateral ankle ligament injury confirmed on ultrasound or MRI and injuries had to be less than six weeks old. Ankle instability was confirmed by the treating author by positive drawer test and positive varus instability in theatre under anaesthesia. Patients excluded from the study were patients with chronic injuries, syndesmosis injuries or any ankle fractures that required fixation or immobilisation or any patients requiring secondary procedures.

General demographic data was collected as well as post-surgical rehabilitation and return to activity. The patient reported outcomes used were the RAND SF- $36^{1-3}$ and the AOFAS score. ${ }^{4}$ The AOFAS questionnaire measures pain (40 points), function (50 points) and 
alignment (10 points, total of 100 points) objectively and subjectively in three independent categories to answered by both the patient and physician, therefore not a patient related outcome measure (PROM). As the AOFAS is a PROM, it has standardized assessment from patients with foot an ankle disorders making it a preferred and widely used measurement tool although not validated. ${ }^{5}$ The RAND SF-36 scale, however, unlike the AOFAS it is a self-administered PROM. It was composed to survey the health status in the medical outcomes study (SF-36). ${ }^{14}$ Each scale is represented as a percentage value.

The data was collected by the researcher, by reviewing the patient files. The PROM's and questionnaires were sent electronically via email to patients to complete and if patients weren't able to complete it electronically, they could do it personally at the follow up date when the functional outcomes were measured. The functional outcomes were collected by the researcher at an appointed date. The electronic questionnaires were done via google forms which automatically creates a spreadsheet, which was then used for analysis. The functional outcomes and the demographic data were manually captured in excel spreadsheet and from there analysed.

\section{Statistical analysis}

The descriptive statistics mean, median, standard deviation and inter-quartile range were used to describe the functional scores from the RAND SF36, AOFAS, including other continuous variables. Frequencies and proportions were used to describe the categorical variables. Group means for the AOFAS were determined, with correlation using Pearson's correlation on the AOFAS and the Rand SF-36. A survival model was used to determine time to return to work/ activity. All statistical analysis were evaluated using STATA 16 and GraphPad Prism software V6.

\section{Surgical procedure}

The surgical procedure that was followed consisted of direct anatomical repair using the Broström-Gould method via an anterolateral approach just anterior to the fibula and entails direct suturing of the ATFL and or the CFL and strengthens the repair with a mobilized lateral position of the extensor retinaculum. ${ }^{1}$ Augmentation of the modified Broström-Gould ATFL repair is performed with an Internal Brace $^{\mathrm{TM}}$ which consists of fibrewire suture tape and SwiveLock anchor (Figure 3) (Arthrex Inc., Naples FL, USA). ${ }^{6}$

The brace is inserted by placing a tunnel in the body of the talus at the anterior talofibular ligament attachment (an area of non-articulating surface) and is angled 45 degrees posteromedially. An internal brace SwiveLock anchor is inserted with fibrewire suture tape; a second drill hole is then made into the distal fibula, angling it slightly proximal. The ankle is then held in neutral position with haemostat under the suture tape to provide some laxity for normal ankle inversion. The wound is then closed in normal fashion. ${ }^{7}$ Post operatively patients are kept on a non-weight bearing splint for 2 weeks, followed by full weight bearing in a removable boot for another 4 weeks in which ankle mobilisation and rehabilitation of the ankle is started aiming for return to sport or activity $6-12$ weeks post-operative.

\section{Results}

A total of 30 patients were entered for the study. Their mean age was 34.7 years (range between 18 to 68 ) refer to Table 1. Ankle sprain injury was diagnosed in majority of patients ( 28 patients) using ultrasound, with only two patients diagnosed by using MRI scan. Grade II injuries were diagnosed in 19 patients and grade III in the remaining 11 patients. Intraoperatively 23 patients were graded, with
13 being grade II and 10 being grade III, in contrast to 15 preoperative grade II injuries and 8 being grade III injuries. Findings from the two modalities agreed ( $\mathrm{p}=0.7055$, McNamar's test).

Table I Results for demographics and general questionnaire

\begin{tabular}{ll}
\hline \multicolumn{2}{l}{ Results for demographics and general questionnaire } \\
\hline Age [mean (range)] & 34.7 (I8-68) \\
Gender & \\
Male [n] & II \\
Female [n] & 19 \\
Time to surgery [mean (range)] & I7 days (2-4I) \\
Time to evaluation [mean (range)] & 24.88 months (I4.4-37.87) \\
Time to return to activity [mean & 6.6 weeks (5-I4) \\
(range)] & $n: 2$ I \\
Time to previous level of activity & I0.94 weeks (6-24) \\
[mean (range)] & $n: 19$ \\
\hline
\end{tabular}

The mean AOFAS score for this study was $92.7 \pm 10.1$ at final follow up, with $16(53,3 \%)$ patients in the study reporting a maximum score of 100 (Table II). A further $5(16.7 \%)$ also reported excellent results (score between 90-100), 6 (20.0\%) reported good scores (80-89), and $3(10 \%)$ reported fair scores (79-60). This indicates an excellent outcome of the augmented Broström-Gould procedure used within this study. ${ }^{8}$ Following the procedure, patients perceived overall low pain, with average pain score of $35 \pm 6.82$ on the AOFAS, with 18 $(60 \%)$ of patients reporting no pain (maximum score of 40 ), and 12 (40\%) (score of between 10 and 30) reporting marginal pain.

Table 2 Mean AOFAS score

\begin{tabular}{lll}
\hline Measurement & & Mean \pm SD \\
\hline Pain & Activity limitation & $35.0 \pm 6.82$ \\
& Maximum walking distance & $4.7 \pm 0.9$ \\
& Walking surface & $4.4 \pm 1.2$ \\
Functionality & Gait abnormality & $7.8 \pm 0.7$ \\
& Sagittal motion & $8.0 \pm 0.0$ \\
& Hindfoot motion & $6.0 \pm 0.0$ \\
& Ankle-Hindfoot stability & $6.0 \pm 0.0$ \\
& Total functionality & $47.9 \pm 0.7$ \\
Alignment & & $9.8 \pm 0.9$ \\
Total & & $\mathbf{9 2 . 7 \pm 1 0 . 1}$ \\
\hline
\end{tabular}

Patients also reported good functionality with an average score of $47.9 \pm 0.7$ on the AOFAS. Only 8 (26.3\%) patients reported some activity limitation, with an average of $9 \pm 2.7$, and only $4(13.3 \%)$ and $8(26.7 \%)$ of patients perceiving a low influence on maximum walking distance and walking surface (mean of $4.7 \pm 0.9$ and $4.4 \pm 1.2$ ). Only one patient (3\%) indicated impaired gait abnormality (mean of $7.8 \pm 0.7$ ). None of the patients experienced any sagittal, hindfoot and ankle-hindfoot stability limitation (all with maximal scores). Patients also reported little influence on alignment (mean 9.8 \pm 0.9 ), with only one patient $(3 \%)$ indicating poor alignment, patient had mild varus heel deformity with subtalar stiffness which was present prior to injury. 
According to the questionnaire, only $9(30 \%)$ of patients in the study were not actively participating in sport prior to injury. The remaining $21(70 \%)$ of patients in the study participating in sport, $14(46.7 \%)$ participated in amateur and $16(53.3 \%)$ in recreational sporting activities. Most patients $61.9 \%$ mainly participated in solitary sporting activities such as golfing, cycling, hiking/walking, running, swimming, horse riding, aerobics, and gym with $38.1 \%$ participating in team sports such as soccer, rugby, hockey, netball, cricket, and softball. Out of all the patients in the study $28(93.3 \%)$ had some form rehabilitation post-surgery, majority 27 (89.7\%) through a physiotherapist and the remainder from both a biokineticist and physiotherapist. Most patients 22 (78.6\%) completed their rehabilitation in $<6$ weeks. The patient that did not attend physio did not have the financial means to do so or chose not to attend a physiotherapist.

The patients were able to return to activity on average 6.6 weeks after injury and previous level of activity by 10.94 weeks (Table I). Of the $21(70 \%)$ of patients partaking in sporting activities, $20(95 \%)$ of patients were able to return to a lower level of sport/activity, with $13(61.9 \%)$ returning to their previous level of activity within $<12$ weeks. Of the remaining patients, only $10 \%$ reported not being able to eventually return to their previous level of activity within a 15 week to 6-month period. In total, 18 (85.7\%) of patients reported as being able return to their respective sporting actives following the augmented Broström procedure over a 6-month period following rehabilitation. The remainder of the patients who did not return sport at final follow up was either still busy with rehabilitation or elected to forgo their sporting activities. This indicated a good functional outcome of the augmented Broström procedure with suture tape.

As part of the SF-36, patient physical and emotional wellbeing was also assessed following augmented Broström procedure with suture tape (Table III). Patients reported overall low physical limitations at final follow up as indicated with high SF-36 scores $>80.0$ (84.36 \pm 26.06 ) measuring physical aspects (incl. physical functioning, role limitations and pain). However, moderate to high SF-36 scores $76.9 \pm 24.2$ measuring mental and social aspects (role limitations due to emotional problems, energy/fatigue, social functioning, and emotional well-being) were reported. In all, patients considered themselves with fair general health $74.3 \pm 19.7$ following the procedure.

Table 3 Mean SF-36 subscale scores

\begin{tabular}{lll}
\hline SF-36 subscale & & Mean \pm SD \\
\hline Physical aspects & $\begin{array}{l}\text { Physical functioning } \\
\text { Role limitations due to } \\
\text { physical health }\end{array}$ & $88.3 \pm 20.6$ \\
& $\begin{array}{l}\text { Pain } \\
\text { Role limitations due to } \\
\text { emotional problems }\end{array}$ & $80.2 \pm 35.0$ \\
& $\begin{array}{l}\text { Energy/fatigue } \\
\text { Social aspects }\end{array}$ & $63.8 \pm 28.3$ \\
& $\begin{array}{l}\text { Emotional well-being } \\
\text { Social functioning }\end{array}$ & $76.0 \pm 20.9$ \\
& General health & $80.4 \pm 24.5$ \\
& & $74.3 \pm 19.7$
\end{tabular}

Despite well correlating RAND SF-36 data for pain vs physical function $\mathrm{r}=0.7432(p<0.0001)$, the Pearson correlation coefficients between the AOFAS score and SF-36 pain $\mathrm{r}=0.5768(p=0.0008)$ and physical functioning $\mathrm{r}=0.5617$ ( $p=0.0012)$ showed significant weak correlations.
In the patients treated with surgery, one patient developed deep venous thrombosis and was treated with anticoagulation therapy for six months, patient had persistent pain in the affected leg afterwards which was reflected in her pain score scoring 20 out of 40 in the AOFAS and 22.5 out 100 for the RAND SF-36.

\section{Discussion}

Lateral ankle ligament injuries grade II/III are generally treated conservatively, which entails functional rehabilitation. ${ }^{9,10}$ Hubbard et al. in a meta-analysis showed that it took between 6 weeks and 3 months for ligament healing to occur. ${ }^{11}$ Functional rehabilitation generally has good outcomes without the surgical complications, however $10-30 \%$ of patients might have chronic symptoms like instability, synovitis or tendinitis, stiffness or pain, ${ }^{12}$ which was also shown by Hubbard et al. who reported that approximately $30 \%$ of patients still had objective laxity or subjective instability at 6 weeks to 1 years following adequate rehabilitation. ${ }^{11}$

There has been recommendations that grade III injuries especially in higher demand patients like professional athletes should be managed surgically. ${ }^{13,14}$ Patients who have undergone surgical management tend have less recurrent ankle sprains and also tend to report less instability. ${ }^{15}$ This was also found the study of Pihlajamaki although it was not clinically significant. ${ }^{14}$ There is very limited studies available regarding acute ligament repair, with majority of studies reporting on chronic ligament repair.

Patients with grade III lateral ankle instability who have undergone surgical treatment have generally been treated with the Broström procedure or the Broström-Gould procedure. Guelfi et al. did a systematic review of open and arthroscopic Broström or BroströmGould procedures of chronic ankle injuries. A total of 505 patients in 13 studies were treated with an open procedure, this included 2 studies on the original Broström procedure, 3 studies using the BroströmGould procedure, 2 studies using the Karlsson procedure and 6 studies using the Broström-Gould procedure with suture anchors. The average AOFAS score was 90.1 and return to sport 4.7 months (18.8 weeks). ${ }^{16}$ However, studies showed that a Broström repaired ligament is significantly weaker than the native uninjured ATFL ligament, ${ }^{17}$ which leads to procedural failure under unprotected motion, thus patients are immobilised or protected for longer. ${ }^{18}$ This leads to lower return rates to previous activity and eventual poor functional outcomes in patients. ${ }^{19}$ Therefore, in an aim of improving procedural robustness and long-term sustained ankle stability and eventual patient outcome, augmentation of the Broström procedure using suture tape has been implemented. ${ }^{20}$ The majority of the literature regarding suture tape augmentation is on chronic ankle ligament injuries, with limited reports on its use in the acute setting. Several reports have demonstrated that this modification notably improves and protects the lateral ligament repair, ${ }^{21,22}$ and maintained ankle stability ${ }_{,}^{20}$ and a lowered risk of instability with recurrent ankle inversion. ${ }^{6,23}$

In our study the post-operative patient outcome of ankle sprain using the modified Broström procedure augmented with suture tape was evaluated using AOFAS and SF-36 scales in 30 patients at final follow up of 24.88 months (range 14.4 - 37.87). Overall, postoperative patient evaluation showed excellent functional outcomes with an AOFAS score of $92.7 \pm 10.1$. This outcome is comparable to similar studies which also evaluated physical outcomes of the modified Broström procedure augmented with suture tape. ${ }^{6,23}$

There are very limited studies regarding acute lateral ankle ligament repair with suture tape augmentation, with the majority of 
studies focusing on chronic lateral ankle ligament repair. A previous study performing only the Broström procedure on 73 patients with chronic injuries over an evaluation period of 64 months showed an AOFAS of 95, which is comparable to the scores obtained in this and other studies. However, it should be noted that $17 \%$ of the patients in that study reported functional instability, which was not reflected in the AOFAS score. ${ }^{24}$

When evaluating return to sport in our study $95 \%$ of patients returned to lower level of activity by 6.6 weeks (range $5-14$ ). When evaluating return to previous level of activity $61.9 \%$ of patients returned to sport in less than 12 weeks i.e.10.94 weeks (range 6-24) and by 6 -months $85.7 \%$ of the patients returned to their previous level of activity after rehabilitation. When comparing it to the study by White et al. where they reported a return to training/activity of 9 weeks (range 7-21.7) and return to play/previous level of activity of 11 weeks (range 8-25.4), the findings is similar but the augmentation with suture tape has approximately 2 weeks earlier return to training. ${ }^{25}$

Previous reports of Broström-Gould procedure reported that only $25-60 \%$ of patients were able to return to previous activity levels over a 6-month period. ${ }^{26}$ Brodsky et al. which performed Broström-Gould procedures in chronic lateral ankle ligament instability reported overall mean physical aspect scores of $84 \%$ compared to $84.4 \%$ in our study, with no correlation observed between AOFAS and SF-36 scores $\mathrm{r}=0.29$ in their study. ${ }^{24}$

When evaluating lateral ligament repair using Broström-Gould with suture tape augmentation Coetzee et al. ${ }^{6}$ found AOFAS score of $94.3 \pm 9.3$ which is similar to our findings and a return to sport rate within a mean of 84 days (12 weeks), which is roughly what we found in our study. In their study the grading and the activity level of the patients weren't mentioned.

When reviewing complications due to surgery, Guelfi et al. in their systematic review of chronic ligament repairs found complication rates of $40(7.92 \%)$ in 505 patients, of which $10(1.98 \%)$ was superficial wound problems. ${ }^{16}$ Due to the paucity of literature regarding suture tape augmentation especially with regards to acute injuries we have to draw some analogies from chronic ligament repair with suture tape augmentation. In the study of Cho et al. they reported a complication rate of $4(13.33 \%)$ out of 30 patients in revision surgery with suture tape augmentation. When reviewing primary suture tape augmentation in chronic ankle ligament repair Ramirez-Gomez et al found a complication rate of $2(7.14 \%)$ in 28 patients. ${ }^{23}$ In the study by Coetzee et al. $2(2.46 \%)$ out of 81 developed superficial wound problems, which is comparable to the non-augmented open procedures mentioned in Guelfi et al's study. Our study had one (3.33\%) patient developing a deep venous thrombosis, but the exclusion of secondary procedures might have had a bias to exclude other complications.

When reviewing the outcomes of this study and the few other similar studies, augmentation may be a good option when surgery is considered especially in athletic patients that will lead to earlier return to sport and activity.

\section{Limitations}

The study consisted of a small sample size. Patients who undergone secondary surgeries were excluded thus safety could not be determined. Inclusion of possible grade II injuries could skew data in a favourable manner.

\section{Conclusion}

Therefore, in conclusion due to the shorter time required for patients to return to lower level and previous levels of activity, this study shows good functional outcome and return to sport levels in patients with lateral ankle ligament injuries that had undergone Broström-Gould procedure augmented with suture tape.

\section{Ethical statement}

The author/s declare that this submission is in accordance with the principles laid down by the Responsible Research Publication Position Statements as developed at the 2nd World Conference on Research Integrity in Singapore, 2010.

Ethical approval was obtained from the research ethics committee health faculty of the University of Pretoria, 88/2019. All procedures were in accordance with the ethical standards of the responsible committee on human experimentation (institutional and national) and with the Helsinki Declaration of 1975, as revised in 2008. Informed written consent was obtained from all patients for being included in the study.

\section{Author contributions}

LWS: Primary author, study design, data collection, data analysis

JW: Primary surgeon, conceptualisation, manuscript revision

CJVR: Statistical analysis

$\mathrm{MN}$ : Conceptualisation, study design, manuscript revision, and supervision.

\section{Acknowledgments}

None.

\section{Funding sources}

No funding was received for this study.

\section{Conflicts of interest}

The author declares that there are no conflicts of interest.

\section{References}

1. Patel AAMD, Donegan DMD, Albert TMD. The 36-Item Short Form. Journal of the American Academy of Orthopaedic Surgeons. 2007;15(2):126-134.

2. Ware JE Jr, Sherbourne CD. The MOS 36-item short-form health survey (SF-36). I. Conceptual framework and item selection. Medical care. 1992;30(6):473-483.

3. Hays RD, Morales LS. The RAND-36 measure of health-related quality of life. Ann Med. 2001;33(5):350-357.

4. SooHoo NF, Vyas R, Samimi D. Responsiveness of the foot function index, AOFAS clinical rating systems, and SF-36 after foot and ankle surgery. Foot \& ankle international. 2006;27(11):930-934.

5. Pena F, Agel J, Coetzee JC. Comparison of the MFA to the AOFAS Outcome Tool in a Population Undergoing Total Ankle Replacement. Foot \& ankle international. 2007;28(7):788-793.

6. Coetzee JC, Ellington JK, Ronan JA, et al. Functional Results of Open Broström Ankle Ligament Repair Augmented With a Suture Tape. Foot \& ankle international. 2018;39(3):304-310.

7. Mackay GMBFFMD, Ribbans WJPFF. The Addition of an "Internal Brace" to Augment the Brostrom Technique for Lateral Ankle Ligament Instability. Techniques in Foot \& Ankle Surgery. 2016;15(1):47-456.

8. Ceccarelli F, Calderazzi F, Pedrazzi G. Is There a Relation between AOFAS Ankle-Hindfoot Score and SF-36 in Evaluation of Achilles Ruptures Treated by Percutaneous Technique? The Journal of Foot and Ankle Surgery. 2014;53(1):16-21. 
9. Taghavi CE, SooHoo NF. Lateral ankle instability. Current Orthopaedic Practice. 2009;20(2):117-122.

10. van den Bekerom MP, Kerkhoffs GM, et al. Management of acute latera ankle ligament injury in the athlete. Knee surgery, sports traumatology, arthroscopy: official journal of the ESSKA. 2013;21(6):1390-1395.

11. Hubbard TJ, Hicks-Little CA. Ankle ligament healing after an acute ankle sprain: an evidence-based approach. Journal of athletic training. 2008;43(5):523-529.

12. Lynch SA, Renstrom PA. Treatment of acute lateral ankle ligament rupture in the athlete. Conservative versus surgical treatment. Sports medicine (Auckland, NZ). 1999;27(1):61-71.

13. Ferran NAMM, Oliva FMDP, Maffulli NPF. Ankle Instability. Sports Medicine \& Arthroscopy Review. 2009;17(2):139-145.

14. Pihlajamaki H, Hietaniemi K, Paavola M, et al. Surgical versus functional treatment for acute ruptures of the lateral ligament complex of the ankle in young men: a randomized controlled trial. The Journal of bone and joint surgery American volume. 2010;92(14):2367-2374.

15. Kerkhoffs GM, Handoll HH, de Bie R, et al. Surgical versus conservative treatment for acute injuries of the lateral ligament complex of the ankle in adults. The Cochrane database of systematic reviews. 2007(2):Cd000380.

16. Guelfi M, Zamperetti M, Pantalone A, et al. Open and arthroscopic lateral ligament repair for treatment of chronic ankle instability: A systematic review. Foot Ankle Surg. 2018;24(1):11-18.

17. Waldrop NE, 3rd, Wijdicks CA, Jansson KS, et al. Anatomic suture anchor versus the Broström technique for anterior talofibular ligament repair: a biomechanical comparison. The American journal of sports medicine. 2012;40(11):2590-2596.

18. Kirk KL, Campbell JT, Guyton GP, et al. ATFL elongation after Brostrom procedure: a biomechanical investigation. Foot \& ankle international. 2008;29(11):1126-1130.
19. Maffulli N, Del Buono A, Maffulli GD, et al. Isolated anterior talofibular ligament Broström repair for chronic lateral ankle instability: 9-year follow-up. The American journal of sports medicine. 2013;41(4):858864.

20. Cho BK, Kim YM, Choi SM, et al. Revision anatomical reconstruction of the lateral ligaments of the ankle augmented with suture tape for patients with a failed Broström procedure. The Bone \& Joint Journal. 2017;99B(9):1183-1189.

21. Schuh R, Benca E, Willegger $M$, et al. Comparison of Broström technique, suture anchor repair, and tape augmentation for reconstruction of the anterior talofibular ligament. Knee Surgery, Sports Traumatology, Arthroscopy. 2016;24(4):1101-1107.

22. Willegger M, Benca E, Hirtler L, et al. Biomechanical stability of tape augmentation for anterior talofibular ligament (ATFL) repair compared to the native ATFL. Knee surgery, sports traumatology, arthroscopy: official journal of the ESSKA. 2016;24(4):1015-1021.

23. Ramírez-Gómez VJ, Gómez-Carlín LA, Ortega-Orozco R, et al. Clinical and Functional Results of Broström-Gould Procedure With Suture Tape Augmentation: An Evaluation Using Three Scales. The Journal of Foot and Ankle Surgery. 2020;59(4):733-738

24. Brodsky AR, O’Malley MJ, Bohne WH, et al. An analysis of outcome measures following the Broström-Gould procedure for chronic lateral ankle instability. Foot \& ankle international. 2005;26(10):816-819.

25. White WJ, McCollum GA, Calder JD. Return to sport following acute lateral ligament repair of the ankle in professional athletes. Knee surgery, sports traumatology, arthroscopy: official journal of the ESSKA. 2016;24(4):1124-1129.

26. Safran MR, Zachazewski JE, Benedetti RS, et al. Lateral ankle sprains: a comprehensive review part 2: treatment and rehabilitation with an emphasis on the athlete. Medicine and science in sports and exercise. 1999;31(7 Suppl):S438-S447. 